DOI https://doi.org/10.30525/978-9934-26-183-1-19

\title{
CHEMISTRY. POLARON CONDUCTIVITY OF POLIMERS
}

\author{
Terebinska M. I. \\ Candidate of Chemical Sciences, \\ Senior Science Researcher \\ Chuiko Institute of Surface Chemistry \\ of National Academy of Sciences of Ukraine \\ Tkachuk O. I. \\ Candidate of Chemical Sciences, \\ Young Scientist \\ Chuiko Institute of Surface Chemistry \\ of National Academy of Sciences of Ukraine \\ Datsiuk A. M. \\ Candidate of Chemical Sciences, \\ Senior Science Researcher \\ Chuiko Institute of Surface Chemistry \\ of National Academy of Sciences of Ukraine \\ Kyiv, Ukraine
}

Nowadays, polymers with conjugated carbon chains constitute a promising class of compounds to be convertible into conducting polymers (CP) (so-called «synthetic metals») that possess electronic, electric, magnetic and optic properties of metals, while keeping plastic properties of ordinary polymers. Typical low conductivity of original conjugated polymers $\left(10^{-10}\right.$ to $10^{-5} \mathrm{~S} \bullet \mathrm{cm}^{-1}$ ) increases significantly (up to 1 to $10^{5} \mathrm{~S} \bullet \mathrm{cm}^{-1}$ ) upon adding small amounts of specific admixtures (dopants) in their matrix. One performs such a doping by either chemical / electrochemical oxidation ( $p$ doping) or reduction ( $n$ doping). As the result, polymer chains acquire positive and negative charges, respectively; the latter are compensated by an incorporation of opposite-sign charges from an electrolyte solution into a polymer matrix. By fine-tuning the doping extent, one can vary the electric conductivity of a $\mathrm{CP}$ in a wide range.

An investigation of a $\mathrm{CP}$ is mainly reduced to clarifying the nature and properties of their charge carriers that usually consist of polarons or / and bipolarons with or without spin. Studies of this kind can crucially facilitate the 
development of new CP-based materials with improved properties for their subsequent application in molecular electronics.

The most extensively studied CPs are polythiophenes, in particular, poly(3,4-ethylenedioxythiopehene). However, even the latter lacks a reliable information on the nature of its charge carriers and their dependence on the polymer-chain length.

In this work we apply the Kohn-Sham density functional theory (B3LYP / 6-31G**) to study the electronic and spatial structure of the 3,4-ethylenedioxythiopehene oligomer consisting of its 12 units (E12), in its various charge states: $0,+1,+2,+3$, and +4 . The doping extent is thus simulated by the charge increase of the corresponding oligomer species. We employed spin-unrestricted calculations (UB3LYP) for charge states +1 and +3 .

Calculated $\mathrm{C}-\mathrm{C}$ bond length between neighboring monomeric units amounts $1.433 \AA$ for the electroneutral state of E12 that is typical for a benzenoid phase (Fig.1). However, even an insignificant extent of doping (being simulated by the cation $\mathrm{E} 12^{+1}$ ) leads to a monotonous shortening those bonds from the ends of the chain to its center with the minimal value of 1.417 $\AA$ at bond 6; the latter being typical for a quinoid structure. The dication E12 $2^{+2}$ shows similar behavior of the abovementioned bond lengths. However, the latter are noticeably smaller in comparison with the similar bond lengths in E12 ${ }^{+1}$, thus showing an increase of the quinoid-phase contribution. Higher oxidation states $(+3$ and +4$)$ show two minima near bonds 2 and 3 , as well as 9 and 10 (Fig.1) that evidences in favor of a further increase of the quinoidphase contribution as well as a formation of two separated polarons at the ends of the oligomer chain.

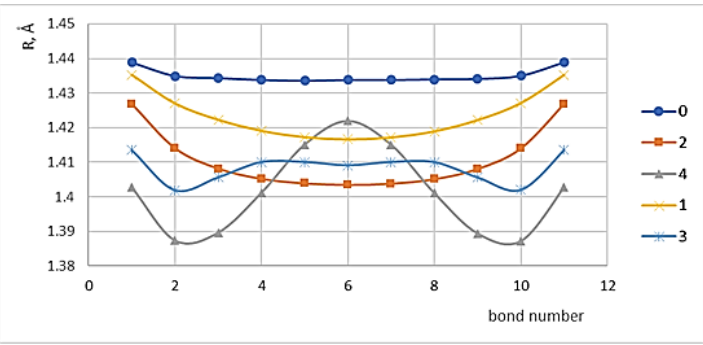

Fig. 1. Dependence of intermonomeric $\mathrm{C}-\mathrm{C}$ bond lengths on the charge state of the E12 oligomer. The bonds are enumerated starting from that between the first and second monomeric units 
The HOMO-LUMO gap in the neutral E12 amounts $2.06 \mathrm{eV}$. As the positive charge (as a model for an increasing doping extent) grows, we observe a progressive appearance of one $\left(\mathrm{E} 12^{+1}\right)$, two $\left(\mathrm{E} 12^{+2}\right)$, three $\left(\mathrm{E} 12^{+3}\right)$, and four $\left(\mathrm{E} 12^{+4}\right)$ polaron levels within the abovementioned HOMO-LUMO gap. A single polaron level in $\mathrm{E} 12^{+1}$ is separated from the top of the valence band by $0.54 \mathrm{eV}$. Two polaron levels in $\mathrm{E} 12^{+2}$ are separated from the valence and conduction bands by $0.50 \mathrm{eV}$. In the cation E12+3, two polaron levels are separated from the top of the valence band by $0.8 \mathrm{eV}$, and the third level is situated $0.90 \mathrm{eV}$ below the bottom of the conduction band. Finally, four polaron levels of $\mathrm{E} 12^{+4}$, build pairwise a prototype of the polaron band within the HOMO-LUMO gap. To demonstrate the formation of two polarons at the ends of the oligomer chain, we show the structure of the molecular orbital that corresponds to one of such levels (Fig.2).

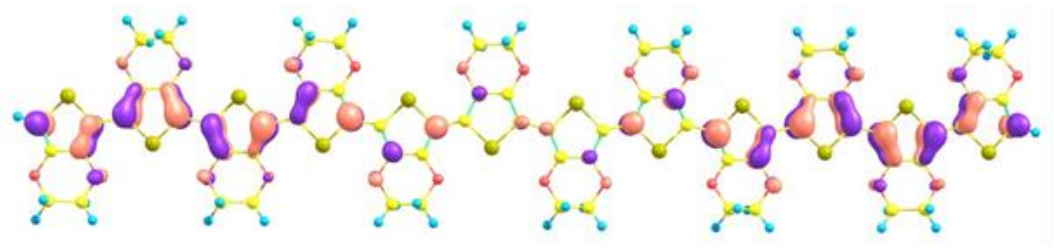

Fig. 2. Structure of the molecular orbital corresponding to the polaron level within the HOMO-LUMO gap of the oligomer $\mathrm{E}^{2+4}$.

Thus, according to our model, the conductivity of a highly doped (oxidized) 3,4-ethylenedioxythiopehene oligomer consisting of its 12 units is provided by two polarons situated at the ends of the chain. For other oligomers, as well as for poly(3,4-ethylenedioxythiopehene), more sophisticated polaron structures might act as charge carriers.

\section{References:}

1. G.M.E. Silva, Electric-field effects on the competition between polarons and bipolarons in conjugated polymers. Phys. Rev. B 61 (2000) P. 10777-10781.

2. S.S. Zade, M. Bendikov, Theoretical study of long oligothiophene dications: bipolaron vs polaron pair vs triplet state. J. Phys. Chem. B 110 (2006) P. 15839- 15846.

3. N. Zamoshchik, U. Salzner, M. Bendikov, Nature of charge carriers in long doped oligothiophenes: the effect of counterions. J. Phys. Chem. C 112 (2008) P. 8408-8418. 